\title{
Comparison of OFDM System in Terms of BER using Different Transform and Channel Coding
}

\author{
Pratima Manhas ${ }^{\mathrm{a}}$, Dr M.K Soni ${ }^{\mathrm{b}}$ \\ ${ }^{a}$ Research Scholar, FET, ECE Deptt, \\ ${ }^{b}$ ED\& Dean, FET, \\ ${ }^{a, b}$ Manav Rachna International University, Faridabad
}

\begin{abstract}
Orthogonal frequency division multiplexing is a type of multicarrier modulation technique which is used in wireless communication. OFDM can be used in various wireless and wired application such Digital audio broadcasting, digital video broadcasting \& wireless LAN. The Performance of OFDM system depends on various parameters such as using different transform, different modulation and by putting various channel coding. In this paper OFDM system is modeled by using different transform (DWT/FFT), different modulation (BPSK, QPSK, QAM) and different channel coding (Linear / Cyclic block codes) for AWGN channel. The performance parameter is calculated as Bit error rate (BER) for various transform/modulation/channel coding based OFDM systems. MATLAB Simulink tool is used to calculate the bit error rate (BER).
\end{abstract}

Index Terms: MATLAB, Transform, Channel coding, wireless LAN, Multicarrier.

(C) 2016 Published by MECS Publisher. Selection and/or peer review under responsibility of the Research Association of Modern Education and Computer Science.

\section{Introduction}

OFDM is a type of multi-carrier modulation (MCM) technique which transmits signals through multiple carriers. These multiple carriers (subcarriers) have different frequencies and all subcarriers are orthogonal to each other [5]. It is used in both wired (ADSL) and wireless communications (wireless LAN) [1].OFDM system has less interference because of the presence of orthogonal subcarriers. So OFDM system is widely used in wireless communication.

\footnotetext{
* Corresponding author.

E-mail address: pratimamehak@gmail.com,ed.fet@mriu.edu.in
} 


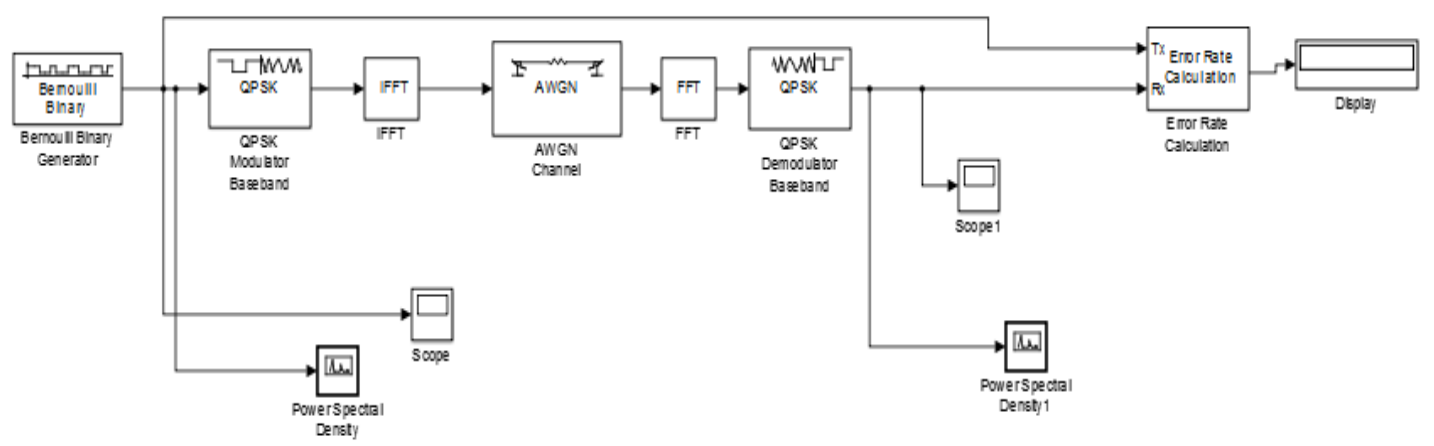

Fig.1. OFDM Simulink Model

The block diagram of OFDM model is shown in Figure 1.In this figure 1, the serial data from the source is first converted into parallel form and then the modulation (mapping) technique is done. After mapping technique, the IFFT (Inverse Fast Fourier Transform) operation is performed to convert frequency domain signal into time domain and then the signal is passed to channel and then at the receiver end the FFT (Fast Fourier Transform) operation is performed to convert back the time domain signal into frequency domain and then demodulation is done and the recovered signal is obtained after passing it to parallel to serial converter [2].

\subsection{OFDM Performance Parameters}

OFDM is very popular technique as it produces very high data rate and which is used in wireless communication system. The performance of OFDM can be measured in interms of Bit error rate(BER).Bit error rate is defined as the ratio of number of errors that occur during transmission to the total number of bits transmitted.

Bit Error rate= Number of Errors occurred during transmission/Total number of bits transmitted

OFDM system can be modeled using various transform, different modulation and channel coding. The behavior of OFDM system using these parameters is evaluated interms of BER.OFDM system is modeled using:

\section{1) DWT/FFT transform}

Wavelet transform are popular and used for the analysis and compression of signals and images. Discrete wavelet transform are applied to discrete data sets and produce discrete outputs [3].

The fast Fourier transform (FFT) is a discrete Fourier transform algorithm which reduces the number of computations needed for $N_{\text {points from }} 2 N^{2}$ to $2 N \lg N$. An FFT is an algorithm that speeds up the calculation of a DFT. The entire purpose of an FFT is to speed up the calculations.

\section{2) Digital Modulation Techniques}

\section{BPSK}

BPSK stands for Binary Phase shift Key. In this digital modulation two phases are generated for the two bit $(1 / 0)$ information. For the transmission of ' 1 ' bit the phase shift is 0 degree and for ' 0 ' bit transmission, the 
phase shift is 180 degree.

\section{QPSK}

It stands for Quadrature phase shift key .It is a type of Phase shift key in which two bits are send at the same time and two bits represents one symbol and four different phases are generated[4].

\section{QAM}

It stands for Quadrature amplitude modulation. It is a combination of both Amplitude shift key (ASK) and phase shift key (PSK)[5].

\section{3) Channel coding}

\section{Linear Block codes}

Block code is a type of error correcting code that acts on a block of $\mathrm{k}$ bits input data to produce $\mathrm{n}$ bits of output data. It is notated as (n, $\mathrm{k})$. Linear block codes are so named because each code word in the set is a linear combination of a set of generator code words [6].

\section{Cyclic Block codes}

An (n, k) linear code C is called a cyclic code if any cyclic shift of a codeword is another codeword. Cyclic codes are popular because they are effective for error correction/detection and have many algebraic properties that simplify encoding and decoding implementation [7] [8].

\section{Proposed Simulation Model}

Following steps are used to model OFDM system:

1. Bernoulli binary generator is used to pass input signal. Then different channel coding is applied on the input sequence. After passing the input signal through various channel coding, then mapping is done using various modulations (BPSK/QPSK and QAM)[9]

2. Then the mapped output is modeled using different transform (DWT/FFT)

3. The different transformed output is passed through the AWGN channel and then demodulation is done to calculate the BER parameter using Error rate calculation. [17]-[18].

The different model of OFDM based on DWT transform and linear coding is shown below in Figures 2-4.

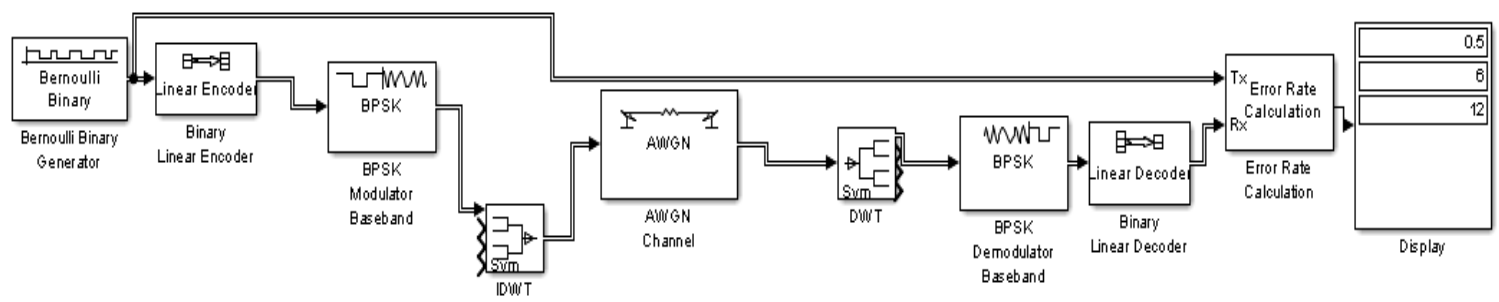

Fig.2. DWT based OFDM model using Linear code with BPSK Modulation 


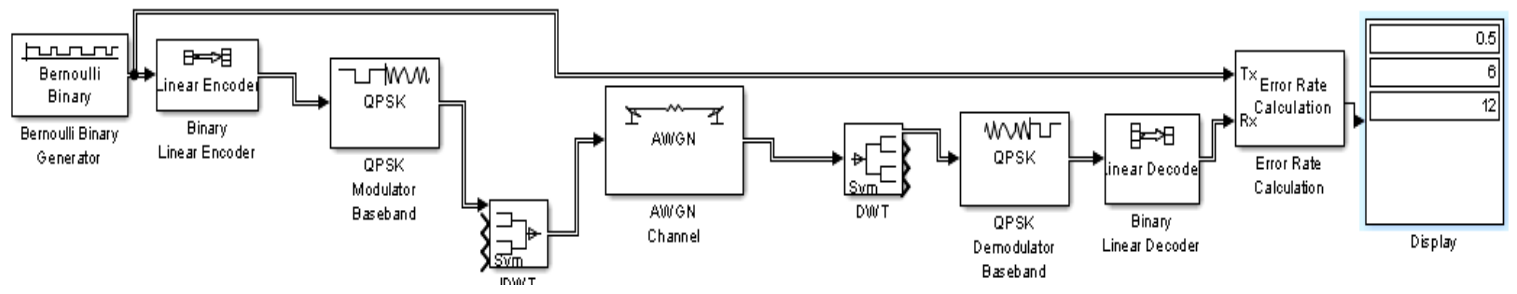

Fig.3. DWT based OFDM Model using Linear Code with QPSK Modulation

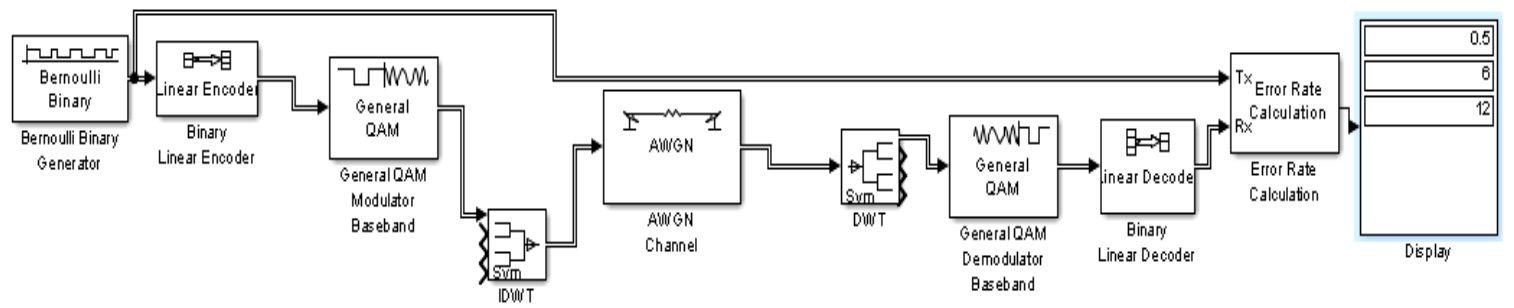

Fig.4. DWT based OFDM Model using Linear Code with QAM Modulation

The different model of OFDM based on DWT transform and cyclic coding for various modulations are shown below in Figures 5-7

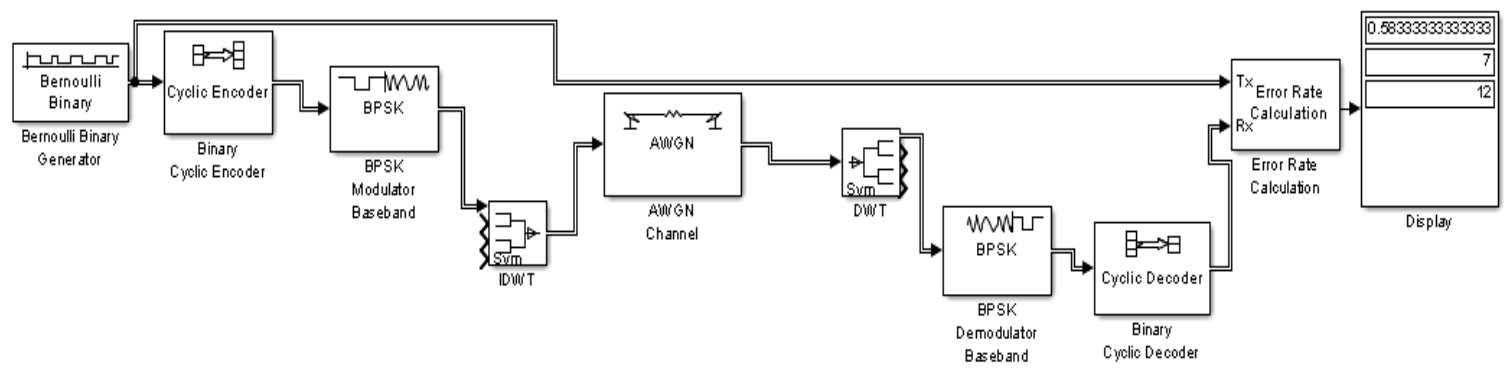

Fig.5. DWT based OFDM Model using Cyclic Code with BPSK Modulation

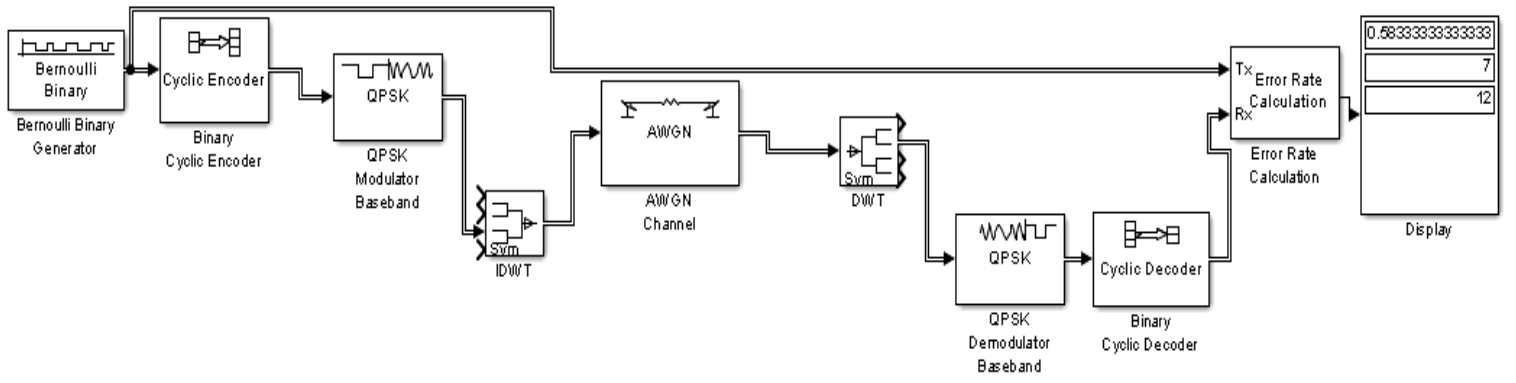

Fig.6. DWT based OFDM Model using Cyclic Code with QAM QPSK Modulation 


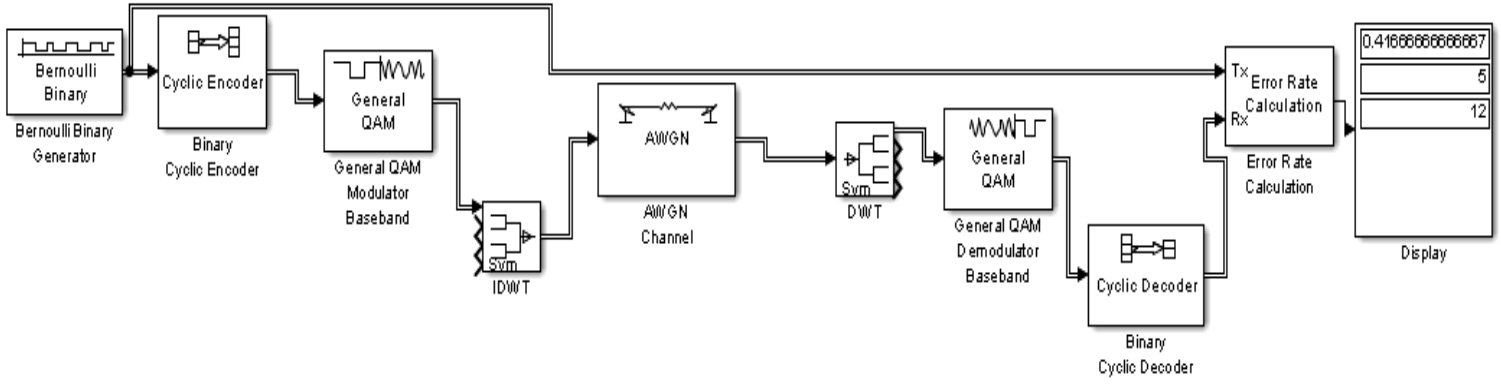

Fig.7. DWT based OFDM Model using Cyclic Code with QAM Modulation

The different model of OFDM using FFT and Linear / cyclic code using QPSK digital modulation are shown in Figures 8-9.

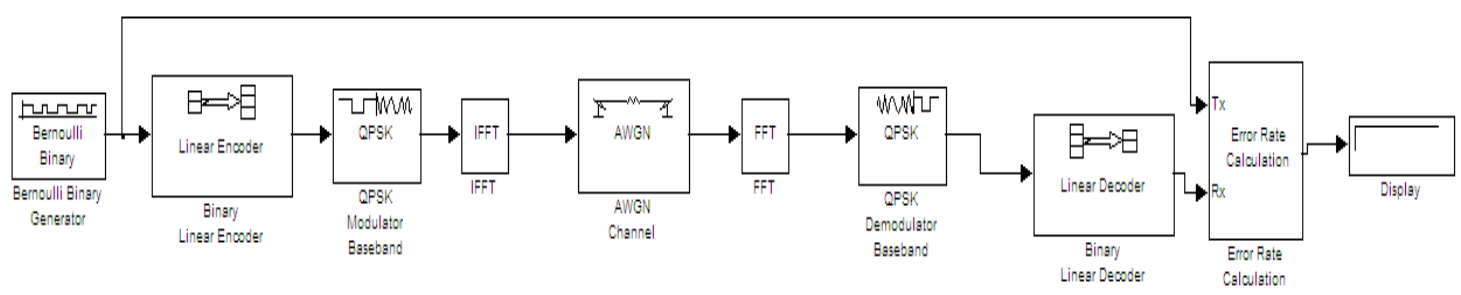

Fig.8. FFT based OFDM Model using Linear Code with QPSK Modulation

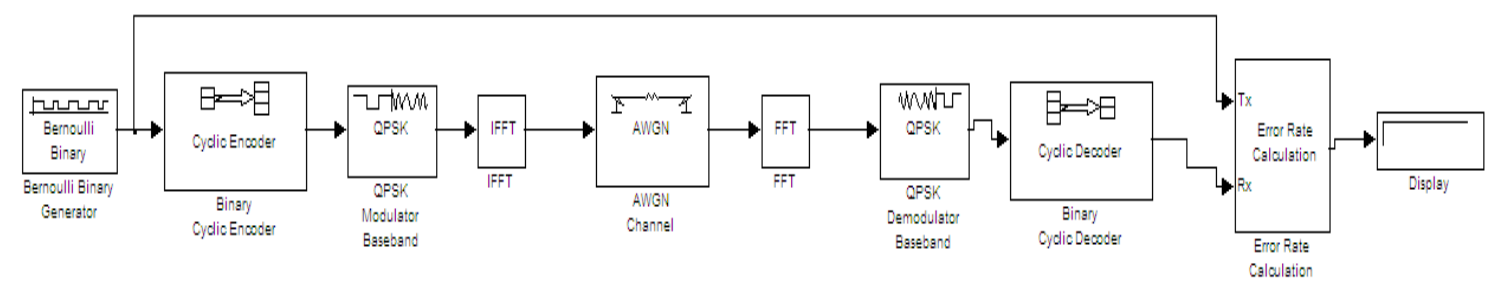

Fig.9. FFT based OFDM Model using Cyclic Code with QPSK Modulation

\section{Simulation Results \& Analysis}

The simulation parameters are as follows:

Transform= DWT, FFT

Modulation = BPSK, QPSK, QAM

Channel coding = Linear block, Cyclic block code

Channel= AWGN

The simulation results is shown in table 1 
Table 1. Simulation results of DWT/FFT based OFDM for Linear/Cyclic codes using BPSK/QPSK/QAM Modulation

\begin{tabular}{|c|c|c|c|c|}
\hline Transform & Coding & BPSK(BER Value) & QPSK(BER value) & QAM(BER value) \\
\hline DWT & Linear & 0.5 & 0.5 & 0.5 \\
\hline DWT & Cyclic & 0.5833 & 0.5833 & 0.4166 \\
\hline FFT & Linear & 0 & 0.0833 & 0.5833 \\
\hline FFT & Cyclic & 0 & 0 & 0.1666 \\
\hline
\end{tabular}

\section{Conclusion}

In today's world there is a requirement for higher data rate which can be used for various applications and for this OFDM system is used. The simulation study calculates the BER for different transform, different modulation and different channel coding. The results shows that FFT based OFDM system produces better results as compared to DWT based OFDM system. Further the performance of OFDM system is better by using cyclic code as compared to Linear code .The OFDM system using Cyclic code and BPSK/QPSK modulation produce minimum value of BER as compared to other modulation technique. So BPSK/QPSK based OFDM system using FFT and cyclic code produces minimum value of BER.

\section{Acknowledgements}

The authors are very much thankful to the reviewers for their comments and suggestions to improve the quality of the manuscript.

\section{References}

[1] Nee, Richard van, and Ramjee Prasad. OFDM for wireless multimedia communications. Artech House, Inc., 2000.

[2] Heiskala, Juha, and John Terry Ph D. OFDM wireless LANs: A theoretical and practical guide. Sams, 2001.

[3] Prasad, Ramjee. OFDM for wireless communications systems. Artech House, 2004.

[4] Joshi, Alok, and Davinder S. Saini. "Performance analysis of coded OFDM for various modulation Schemes in 802.11 a based digital broadcast applications." Information Processing and Management. Springer Berlin Heidelberg, 2010. 60-64.

[5] Jiang, Tao, and Yiyan Wu. "An overview: peak-to-average power ratio reduction techniques for OFDM signals." IEEE transactions on broadcasting 54.2 (2008): 257.

[6] Islam, Mohammed Safiqul, Gouri Rani Barai, and Atiq Mahmood. "Performance analysis of different modulation schemes using OFDM techniques in Rayleigh fading channel." International journal of fundamental Physical sciences 1.1 (2011): 22-27.

[7] Babu, A. Sudhir, and Dr KV Sambasiva Rao. "Evaluation of BER for AWGN, Rayleigh and Rician fading channels under various modulation schemes." International Journal of Computer Applications 26.9 (2011).

[8] Nasir Salh Almisbah, Elessaid S Saad. "Analysis of Intercarrier Interference Cancellation Scheme in OFDM Systems." Communication engineering \& application journal, iaes (2012).

[9] Filbert Hilman Juwono, Randy S. Putra, Dadang Gunawan, ” A Study on Peak-to-Average Power Ratio in DWT-OFDM Systems",iaes (Vol 12, No 5: May 2014). 
[10] Lu, Bao-Liang, Xiaodong Wang, and Ye Geoffrey Li. "Iterative receivers for space-time block-coded OFDM systems in dispersive fading channels." Wireless Communications, IEEE Transactions on 1.2 (2002): 213-225.

[11] Molisch, Andreas F., Aarne Mammela, and Desmond P. Taylor. Wideband wireless digital communication. Prentice hall PTR, 2000.

[12] Hwang, Taewon, et al. "OFDM and its wireless applications: a survey." Vehicular Technology, IEEE Transactions on 58.4 (2009): 1673-1694.

[13] Heiskala, Juha, and John Terry Ph D. OFDM wireless LANs: A theoretical and practical guide. Sams, 2001.

[14] Rong, Yue, Sergiy Vorobyov, and Alex B. Gershman. "Linear block precoding for OFDM systems based on maximization of mean cutoff rate." Signal Processing, IEEE Transactions on 53.12 (2005): 4691-4696.

[15] Debbah, Mérouane. "Short introduction to OFDM." White Paper, Mobile Communications Group, Institut Eurecom (2004).

[16] Daoud, Omar, and Omar Alani. "Peak-to-average power ratio reduction technique for MIMO/OFDM systems." International Journal of Mobile Communications 7.6 (2009): 632-645.

[17] Sanjiv Kumar" BER Analysis of OFDM Digital Communication Systems with Improved ICI Cancellation Technique,IJISA,Vol 6, No. 4, 2014, pp. 56-62.

[18] Sanjiv Kumar, P. K. Gupta, G. Singh and D. S. Chauhan, "Performance analysis of Rayleigh and Rician fading channel models using Matlab simulation” IJISA, vol. 5, no. 09, 2013, pp. 94-102.

\section{Authors' Profiles}

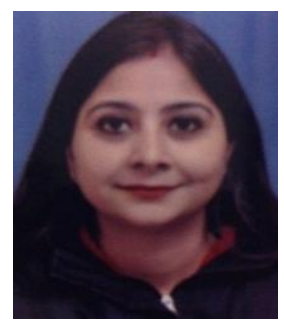

Pratima Manhas is a Research Scholar and doing her research work in the area of Wireless communication (OFDM Systems). She has been associated with teaching from the past 12 years. Currently she is working with Manav Rachna International University Faridabad as an Associate Professor in Electronics \& Communication Engineering Deptt. She had published more than 22 papers in National, International journals and conferences. She had published books on Digital electronics, Microelectronics \& Multimedia systems.

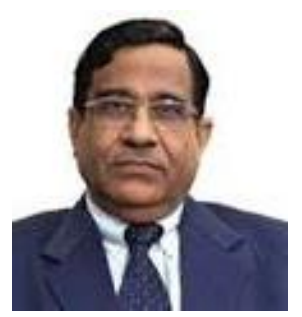

Dr. M. K Soni did his B.Sc (Engg.) in 1972 and M.Sc (Engg.) in 1975 from REC Kurukshetra (Now NIT Kurukshetra) and thereafter completed his Ph.D from REC Kurukshetra (in collaboration with IIT Delhi) in 1988. He has a total 40 years of rich experience into Academics. His area of interest is microprocessor based control systems and digital system design. He has more than 100 research papers in the International and National Journals to his credit. Presently he is Executive Director \& Dean, Faculty of Engineering and Technology, Manav Rachna International University, Faridabad.

How to cite this paper: Pratima Manhas, M.K Soni,"Comparison of OFDM System in Terms of BER using Different Transform and Channel Coding", International Journal of Engineering and Manufacturing(IJEM), Vol.6, No.1, pp.28-34, 2016.DOI: 10.5815/ijem.2016.01.03 